\title{
Simulation-based training following a theoretical lecture enhances the performance of medical students in the interpretation and short-term retention of 20 cross-sectional Transesophageal Echocardiographic views: A prospective, randomized, controlled trial
}

\section{Yang Zhao}

Affiliated Hospital of North Sichuan Medical College

\section{Zong-yi Yuan}

Nanchong Central Hospital

Han-ying Zhang

Pidu district people's hospital

Xue Yang

Children's Hospital of Chongqing Medical University

Duo Qian

Affiliated Hospital of North Sichuan Medical College

Jing-yan Lin

Affiliated Hospital of North Sichuan Medical College

Hai-bo Song

West China Hospital of Sichuan University

Tao Zhu ( $\nabla 739501155 @ q q . c o m$ )

West China Hospital of Sichuan University

\section{Research Article}

Keywords: Echocardiography, Transesophageal, Simulation Training, Retention

Posted Date: February 17th, 2021

DOI: https://doi.org/10.21203/rs.3.rs-200314/v1

License: (c) (1) This work is licensed under a Creative Commons Attribution 4.0 International License. Read Full License 


\section{Abstract}

\section{BACKGROUND}

Both simulation-based training and video-based training are known to serve as educational adjuncts for learning TEE among medical students. In the present study, we hypothesized that simulation-based training would better enhance the performance of medical students in the interpretation of 20 crosssectional views compared to video-based training.

\section{METHODS}

A total of 120 4th -year undergraduate medical students were enrolled in the present study. The study began with a pre-test of all the participants, followed by a 90-min theoretical lecture, after which the participants were required to undertake a post-test. Subsequently, the participants were randomly divided into the video-based group (Group V) and simulation-based group (Group S). Next, Group V received 60 min of TEE video learning, while Group S received 60 min of TEE simulator training. After the respective training, both the groups undertook the retention-test 1 and retention-test 2, one week and one month later, respectively. The overall accuracy demonstrated in each test was used to assess the performance of the participants in the interpretation of 5 views selected randomly from a set of 20 cross-sectional views.

\section{RESULTS}

No statistically significant differences were observed in the overall accuracy in the pre-test and the posttest between the two groups. However, better overall accuracy was observed in Group $S$ in both retentiontest 1 and retention-test 2 compared to Group V.

\section{CONCLUSIONS}

Following a 90-min theoretical lecture, simulation-based training better enhanced the performance of medical students in the interpretation and short-term retention of 20 cross-sectional views compared to video-based training.

\section{Background}

Transesophageal echocardiography (TEE) is considered a valuable and useful tool for the cardiac anatomical and functional evaluation of the patients undergoing surgery, either cardiac or noncardiac[1, 2]. TEE images provide information regarding the cardiac function and the effect of surgical intervention. It is essential for the cardiac anesthesiologists to learn the TEE technique and properly interpret the TEE images in the perioperative period[3]. It is recommended to use 20 cross-sectional views for the basic knowledge of learning TEE[4].

The training methods for learning and interpreting TEE include lectures, simulation-based training, videobased training, actual patient training at the bedside, etc. Generally, hands-on TEE at the bedside is the 
"gold standard", although it requires repetitive exposure to practice with actual patients. A limited number of patients and inadequate practicing time are the two major barriers experienced by the trainees in hands-on TEE[5]. Lectures may assist the trainees in understanding the basic concepts of TEE[6]. Videobased training, when used as an educational adjunct, has been demonstrated to improve the knowledge of interpreting echocardiographic images, although with limited retention[7-9]. Recently, various simulation-based training systems for TEE, cardiac catheterization, coronary angioplasty, etc., have been developed to be used by doctors and for medical education, and the application of these systems has demonstrated improvement in the related procedural capability of the healthcare providers[10]. As an educational adjunct, the TEE simulator training system has been demonstrated to enhance the psychomotor skills in a mannequin by manipulating the probe, thereby improving the ability to interpret echocardiographic TEE images by learning the corresponding cross-sectional images on the screen[11]. However, which one among the two, video-based training and simulation-based training, is better for interpreting 20 cross-sectional views remains unclear. Since the literature recommends a simulationbased training followed by a theoretical lecture[12], we hypothesized that if followed by a theoretical lecture, simulator-based TEE training would improve the performance of novice trainees in the interpretation of 20 cross-sectional views compared to the video-based TEE training.

\section{Methods}

\section{Aim}

The present study aimed to compare the simulator-based TEE training with the video-based TEE training, both following a theoretical lecture, and to determine which of the two would better improve the interpretation of 20 cross-sectional views.

\section{Study design}

The present study was designed as a single-center, randomized, double-blinded, prospective clinical trial. It was conducted from April 2020 to May 2020 in the North Sichuan Medical College, Nanchong City, Sichuan Province, China, and was registered in the Chinese registry of clinical trials at http://www.chictr.org.cn (ChiCTR2000033519; 3/June/2020). The study adhered to the applicable CONSORT guidelines and was approved by the Research Ethics Committee of the Affiliated Hospital of North Sichuan Medical College (approval No. 2020/111-1)

\section{Population}

Informed written consent was obtained from all participants all subjects or, if subjects are under 18 , from a parent and/or legal guardian. All 4th-year medical students with an anesthesia major and without any previous experience in cardiac anesthesia or echocardiography were eligible for participation in the present study. Participation was allowed only after signing the consent form and agreement to adhere to the study requirements. 


\section{Sample Size}

Since no similar previous studies were reported in the literature, the sample size was calculated based on the initial pilot studies, according to which that the mean \pm SD of retention-test 1 was $59.4 \% \pm 21.3 \%$ in the video-based training group comprising ten subjects. With a statistical power of 0.8 and a type 1 error rate of 0.05 in detecting $20 \%$ improvement as conservative, the sample size calculation determined that a minimum of 50 patients were required per group to distinctly present this difference using a two-tailed Student's t-test. Considering a possible dropout rate of $20 \%, 120$ patients were finally included in the present study.

\section{Theoretical lecture}

All the participants together attended a 90-min theoretical lecture, which was taught by a tutor with experience in over 200 cases of TEE image examinations for cardiac surgeries. The lecture entailed the basic concepts of echocardiography, normal cardiac anatomy, a simple explanation of 20 cross-sectional views, and the corresponding anatomical structures.

\section{Randomization}

After the post-test (refer to the assessment section), the participants were randomly divided (sealed envelope by SPSS with random seed 20200611) into the video-based group (Group V) and simulationbased group (Group S) by J.-Y.L. who was not involved in other parts of this study.

\section{Intervention}

The participants belonging to Group V received a 60-min video-based training (30-min of high-definition video-watching under supervision, twice). The video was recorded by Professor Robert L. Lobato, M.D. from Stanford University (refer to the manuscript attachment). The content of the 30-min video comprised: Professor Robert L. interpreting the probe location in each of the 20 cross-sectional views in the PowerPoint, verbally explaining how each of the 20 cross-sectional views formed by depicting the heart model and the corresponding two-dimensional echocardiographic cross-sectional image in the PowerPoint without a visual probe at hand. When the video was playing, each participant had a heart model at hand to understand what was being taught. The tutor also used native language with the participants when appropriate for a better understanding. The students were not allowed to have a discussion with each other and were encouraged to ask the tutor for help if they had any confusion regarding TEE.

Group S was demonstrated the 20 cross-sectional views through the simulator training system for 30 min by the tutor. The training included information regarding how to move the probe to obtain each view, an explanation of the relative position of the heart, and a demonstration of how each sectional image was formed, all of which assisted the trainees in understanding every anatomical structure in the 20 crosssectional views. The participants were allowed to undertake this training only once. Subsequently, each trainee was allowed to manipulate the VirSim TEE simulator training system alone for 30 min under the 
tutor's instructions. The participants were not allowed to watch how their peers manipulated the probe or to have a discussion with each other. Therefore, in total, each participant had $60 \mathrm{~min}$ of training.

The words delivered by the tutors in the 60-min training sessions were almost the same in both the groups, such as 'this image is referred to as middle-esophagus four chambers image', 'when you placed the probe at the middle of the esophagus', 'regulate the scope into zero angle', 'the anatomic structure of the heart marked with the marker are the structures listed on the right part of the screen', 'think, which image have you got', 'so, the images on the screen are: left atrium, left ventricle, right atrium, right ventricle, and so on'.

The participants were requested not to review the related knowledge during the whole period of the trial. After the training sessions, the participants were required to individually complete a checklist, which included the names and the anatomic structures of each of the 20 cross-sectional views. Only those participants who answered all the questions correctly were allowed to undergo the next trial. The participants who could not provide correct answers to all the questions were assisted by the tutor in clarifying their concepts by demonstrating and explaining the heart model for Group $\mathrm{V}$ or by manipulating the simulator for Group S.

\section{Measurements}

Pre-test conducted prior to the training aimed at assessing the pre-existing knowledge of TEE in the participants. After the 90-min lecture, the post-test was conducted to assess the effect of the lecture. Subsequently, the participants were randomly divided into Group V and Group S (with equal participants). After the completion of all the training sessions, the participants were required to undertake the retentiontest 1 conducted one week later and the retention-test 2 conducted one month later.

In all the tests, the participants were shown five slides in PowerPoint, each with an echocardiographic image that was depicted for a maximum of $5 \mathrm{~min}$. The participants were required to select the correct name of the image and the corresponding anatomic structure from a list of options. 20 crosssectional views included : (1) Middle esophagus ascend aortic short axis, ME Asc Aortic SAX; (2) Middle esophagus ascend aortic long axis $\llbracket$ ME Asc Aortic LAX; (3) Middle esophagus descend aortic short axis, ME Desc Aortic SAX; (4) Middle esophagus descend aortic long axis $\llbracket$ ME Desc Aortic LAX; (5)Upper esophagus aortic arch long axis, UE Aortic Arch LAX; (6)Upper esophagus aortic arch short axis, UE Aortic Arch SAX; (7)Middle esophagus four chamber, ME 4C; (8) Middle esophagus mitral commissural, ME MC; (9) Middle esophagus two chamber冈ME 2C; (10)Middle esophagus long axis, ME LAX; (11)Middle esophagus aortic valve short axis $₫$ ME AV SAX; (12)Middle esophagus right ventricle inflow-outflow, ME RVOT;(13) Middle esophagus Bicaval,ME Bicaval; (14)Middle esophagus aortic valve long axis, ME AV LAX; (15) Transgastric basal short axis , TG Basal SAX; (16) Transgastric middle papillary short axis view, TG Mid Papillary SAX; (17) Transgastric two-chamber, TG 2C; (18) Transgastric long axis, TG LAX; (19) Transgastric right ventricle inflow, TG RV inflow; (20) Deep transgastric long axis, Deep TG LAX. Ten anatomic-structure lists included: (1) left atrium, LA (2)left ventricle, LV; (3)mitral valve, MV; (4)aortic valve, AV; (5) aorta, AO; (6) right atrium, RA; (7) right ventricle, RV; (8) tricuspid valve, TV; (9) pulmonary 
valve, PV; (10) pulmonary artery, PA. The overall accuracy, which was defined as the number of correct answers divided by the total number of questions in each test, was used to assess the performance of the participants.

The pre-test included five images, which were generated using SPSS (random seed $=20200610)$ : UE Aortic Arch LAX; ME MC; ME RVOT; TG RV inflow; Deep TG LAX.

The post-test included 5 images, which were again generated using SPSS (random seed $=20200611$ ): ME Desc Aortic LAX; ME 4C; ME LAX; TG LAX; TG RV inflow.

The retention-test 1 included five images generated using SPSS (random seed $=20200618$ ): ME Asc Aortic SAX; ME Desc Aortic SAX; ME 2C; ME RVOT; TG Basal SAX.

The retention-test 2 included five images generated using SPSS (random seed $=20200711$ ): ME DesC Aortic SAX; ME Desc Aortic LAX; ME RVOT; ME AV LAX; TG LAX.

\section{Statistical analysis}

All data were presented as Mean $\pm S D$. A 2-sample t-test was used to evaluate the differences between the group in the pre-test, post-test, retention-test 1 , and retention-test 2 . The paired-sample t-test was used to compare the individual improvement prior to and after the theoretical lecture. The primary outcome was the mean overall accuracy of the retention-test 1 and the secondary outcome was the mean overall accuracy of the pre-test, post-test, and retention-test 2. Statistical significance was determined using the two-tailed test at the P-value threshold of 0.05. All data were analyzed using the SPSS Statistics 25.0 software (Statistical Program for Social Sciences, SPSS Inc, Chicago, Illinois, USA),

\section{Results}

In the period between June 2020 and July 2020, a total of 120 4th -year undergraduate medical students with no previous experience of echocardiography from the North Sichuan Medical college were enrolled in the present study, all of whom completed the retention-test 2 conducted at the end of the trial. The flowchart is presented in Fig. 1, and the demographic data are provided in Table 1. There was no significant difference in terms of age and sex between the two groups (Table 1).

Table 1. Comparison of characteristics between Group V and Group S ( $\pm \mathrm{s})$.

\begin{tabular}{llll}
\hline & Group V $(\mathrm{n}=60)$ & Group $\mathrm{S}(\mathrm{n}=60)$ & P value \\
\hline Sex(male/female) & $19 / 41$ & $23 / 37$ & 0.044 \\
Age(years) & $22.2 \pm 0.8$ & $22.1 \pm 0.9$ & 0.755 \\
& & & \\
\hline
\end{tabular}

Abbreviations: Group V, video-based training group; Group S, simulation-based training. Data presented as the number for sex, mean \pm standard deviation for age. 
Table 2 presents the details of the pre-test, and Table 3 presents the details of the post-test. There were no statistically significant differences in the overall accuracy in the pre-test $(V=8.9 \% \pm 5.2 \%$ vs. $S=9.0 \%$ $\pm 5.2 \%, P=0.885)$ and post-test $(V=47.1 \% \pm 11.2 \%$ vs. $S=46.2 \% \pm 11.0 \%, P=0.682)$ between the two groups. Much improvement in the overall accuracy was observed in the two groups in the post-test compared to the pre-test, which was attributed to the learning from the theoretical lecture (Group V in post-test $=47.1 \% \pm 11.2 \%$ vs. Group V in pre-test $=8.9 \pm 5.2 \%, P<0.0001 ;$ Group $S$ in post-test $=46.2 \%$ $\pm 11.0 \%$ vs. Group $S$ in pre-test $=9.0 \% \pm 5.2 \%, P<0.0001$ ). 
Table 2

Performance of Group V and Group S in the pre-test

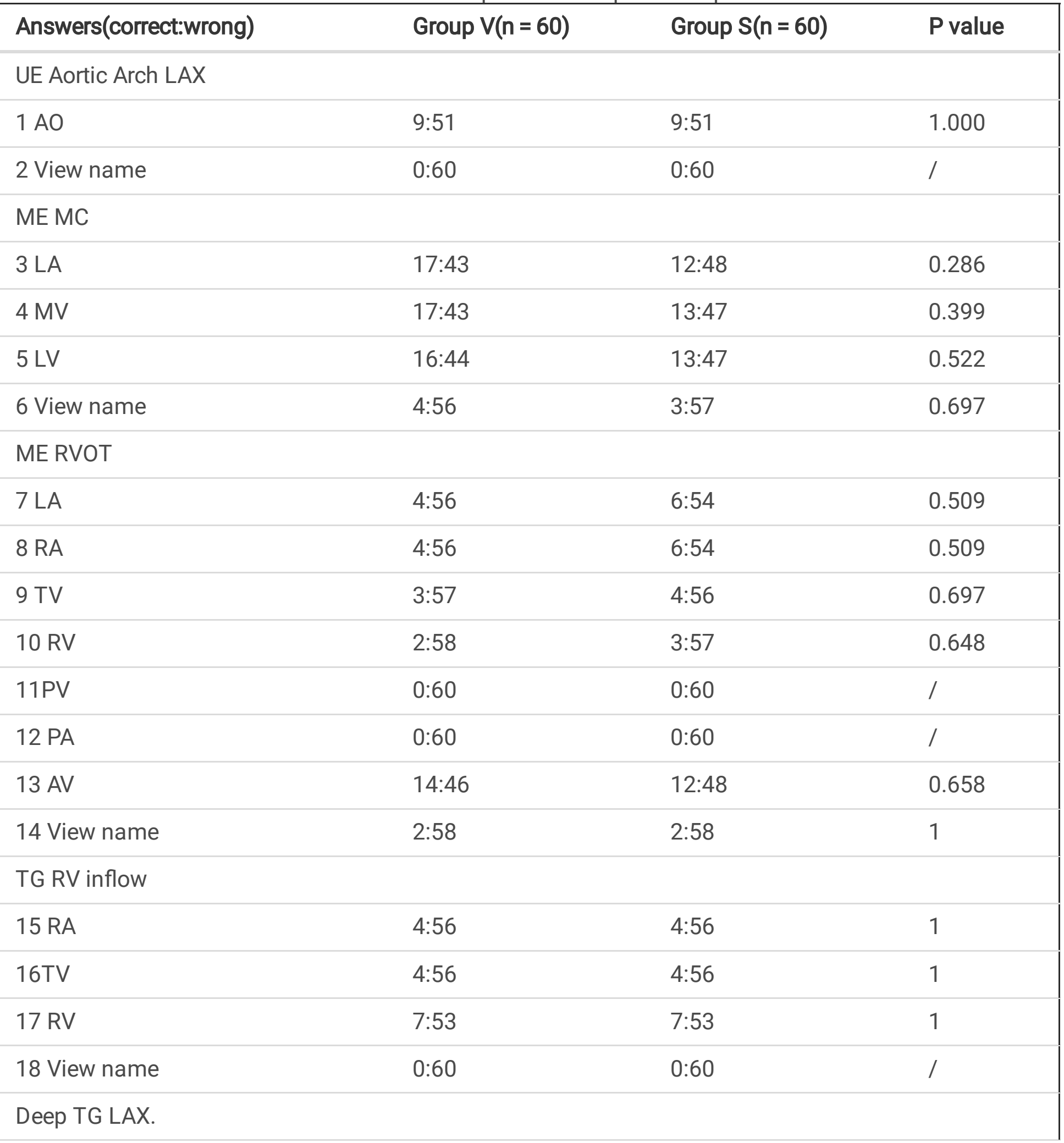

Data presented as the number for the sum of trainees who responded with correct or wrong interpretation of each anatomic structure, mean \pm standard deviation for mean overall accuracy.

Abbreviations: UE Aortic Arch LAX (Upper esophagus aortic arch long axis); ME MC (Middle esophagus mitral commissural); ME RVOT (Middle esophagus right ventricle inflow-outflow); TG RV inflow (Transgastric right ventricle inflow); Deep TG LAX (Deep transgastric long axis). 


\begin{tabular}{|llll|}
\hline Answers(correct:wrong) & Group V( $\mathbf{n}=\mathbf{6 0})$ & Group $\mathbf{S}(\mathbf{n}=60)$ & P value \\
\hline $19 \mathrm{LA}$ & $3: 57$ & $6: 54$ & 0.298 \\
\hline $20 \mathrm{MV}$ & $3: 57$ & $6: 54$ & 0.298 \\
\hline $21 \mathrm{LV}$ & $7: 53$ & $8: 52$ & 0.783 \\
\hline $22 \mathrm{AV}$ & $4: 56$ & $6: 54$ & 0.509 \\
\hline $23 \mathrm{AO}$ & $4: 56$ & $6: 54$ & 0.509 \\
\hline 24 View name & $0: 60$ & $0: 60$ & 0.885 \\
\hline Mean overall accuracy(\%) & $8.9 \pm 5.2$ & $9.0 \pm 5.2$ & $/$ \\
\hline $\begin{array}{l}\text { Data presented as the number for the sum of trainees who responded with correct or wrong } \\
\text { interpretation of each anatomic structure, mean } \pm \text { standard deviation for mean overall accuracy. }\end{array}$ \\
\hline $\begin{array}{l}\text { Abbreviations: UE Aortic Arch LAX (Upper esophagus aortic arch long axis); ME MC (Middle } \\
\text { esophagus mitral commissural); ME RVOT (Middle esophagus right ventricle inflow-outflow); TG RV } \\
\text { inflow (Transgastric right ventricle inflow); Deep TG LAX (Deep transgastric long axis). }\end{array}$ \\
\hline
\end{tabular}


Table 3

Performance of Group V and Group S in the post-test

\begin{tabular}{|c|c|c|c|}
\hline Answers(correct:wrong) & Group V(n=60) & Group $S(n=60)$ & $P$ value \\
\hline \multicolumn{4}{|l|}{ ME Desc Aortic LAX } \\
\hline $1 \mathrm{AO}$ & $42: 18$ & $37: 23$ & 0.336 \\
\hline 2View name & $13: 47$ & $15: 45$ & 0.666 \\
\hline \multicolumn{4}{|l|}{ ME 4C } \\
\hline 3RA & $58: 2$ & $55: 5$ & 0.243 \\
\hline 4TV & $58: 2$ & $56: 4$ & 0.402 \\
\hline $5 \mathrm{RV}$ & $58: 2$ & $57: 3$ & 0.648 \\
\hline $6 \mathrm{LA}$ & $55: 5$ & $52: 8$ & 0.378 \\
\hline $7 \mathrm{MV}$ & $58: 2$ & $59: 1$ & 0.559 \\
\hline 8LV & $59: 1$ & $56: 4$ & 0.171 \\
\hline 9View name & $58: 2$ & $57: 3$ & 0.648 \\
\hline \multicolumn{4}{|l|}{ ME LAX } \\
\hline 10LA & $43: 17$ & $35: 25$ & 0.126 \\
\hline $11 \mathrm{MV}$ & $33: 27$ & $26: 34$ & 0.201 \\
\hline $12 \mathrm{LV}$ & $26: 34$ & $29: 31$ & 0.583 \\
\hline 13AV & $32: 28$ & $37: 23$ & 0.356 \\
\hline $14 \mathrm{AO}$ & $32: 28$ & $37: 23$ & 0.356 \\
\hline $15 \mathrm{RV}$ & $26: 34$ & $29: 31$ & 0.583 \\
\hline 16View name & $27: 33$ & $21: 39$ & 0.264 \\
\hline \multicolumn{4}{|l|}{ TG LAX } \\
\hline 17LA & $12: 48$ & $11: 49$ & 0.817 \\
\hline $18 \mathrm{MV}$ & $5: 55$ & $5: 55$ & 1 \\
\hline $19 L V$ & $12: 48$ & $18: 42$ & 0.206 \\
\hline
\end{tabular}

Data presented as the number for the sum of trainees who responded with correct or wrong interpretation of each anatomic structure, mean \pm standard deviation for the mean overall accuracy.

Abbreviations: ME Desc Aortic LAX (Middle esophagus descend aortic long axis); ME 4C (Middle esophagus four chamber); ME LAX (Middle esophagus long axis); TG LAX (Transgastric long axis); TG RV inflow (Transgastric right ventricle inflow). 


\begin{tabular}{|c|c|c|c|}
\hline Answers(correct:wrong) & Group V(n=60) & Group $S(n=60)$ & $P$ value \\
\hline $20 \mathrm{AV}$ & $0: 60$ & $0: 60$ & / \\
\hline $21 \mathrm{AO}$ & $0: 60$ & $0: 60$ & / \\
\hline 22View name & $11: 49$ & $10: 50$ & 0.810 \\
\hline \multicolumn{4}{|l|}{ TG RV inflow } \\
\hline 23RA & $4: 56$ & $5: 55$ & 0.729 \\
\hline 24TV & $4: 56$ & $5: 55$ & 0.729 \\
\hline $25 \mathrm{RV}$ & $4: 56$ & $5: 55$ & 0.729 \\
\hline 26 View name & $4: 56$ & $4: 56$ & 1 \\
\hline Mean overall accuracy(\%) & $47.1 \pm 11.2$ & $46.2 \pm 11.0$ & 0.682 \\
\hline \multicolumn{4}{|c|}{$\begin{array}{l}\text { Data presented as the number for the sum of trainees who responded with correct or wrong } \\
\text { interpretation of each anatomic structure, mean } \pm \text { standard deviation for the mean overall accuracy. }\end{array}$} \\
\hline \multicolumn{4}{|c|}{$\begin{array}{l}\text { Abbreviations: ME Desc Aortic LAX (Middle esophagus descend aortic long axis); ME 4C (Middle } \\
\text { esophagus four chamber); ME LAX (Middle esophagus long axis); TG LAX (Transgastric long axis); } \\
\text { TG RV inflow (Transgastric right ventricle inflow). }\end{array}$} \\
\hline
\end{tabular}

Better overall accuracy was observed in Group $S$ in retention-test 1 (details presented in Table 4; Group V $=65.2 \% \pm 13.6 \%$ vs. Group $S=83.9 \% \pm 16.5 \%, P<0.0001$ ) and retention-test 2 (details presented in Table 5; Group V $=58.5 \% \pm 13.0 \%, P<0.0001$ vs. Group $S=71.5 \% \pm 10.9 \%, P<0.0001$ ) compared to Group $\mathrm{V}$. The performance in the retention-test 2 was lower than the performance in the retention-test 1 (Group V in retention-test $2=58.5 \% \pm 13.0 \%$ vs. Group $V$ in retention-test $1=65.2 \% \pm 13.6 \%, P=0.007$; Group $S$ in retention-test $2=71.5 \% \pm 10.9 \%$ vs. Group $S$ in retention-test $1=83.9 \% \pm 16.5 \%, P<0.0001)$. The comparison of the two groups in all the tests is presented in Fig. 2. 
Table 4

Performance of Group V and Group S in the retention test 1.

\begin{tabular}{|c|c|c|c|}
\hline Answers(correct:wrong) & Group V(n=60) & Group $S(n=60)$ & $P$ value \\
\hline \multicolumn{4}{|l|}{ ME Asc Aortic SAX } \\
\hline $1 \mathrm{PA}$ & $26: 34$ & 50: 10 & $<0.0001$ \\
\hline $2 \mathrm{AO}$ & 27: 33 & 50: 10 & $<0.0001$ \\
\hline 3 View name & $21: 39$ & 48: 12 & $<0.0001$ \\
\hline \multicolumn{4}{|l|}{ ME Desc Aortic SAX } \\
\hline $4 \mathrm{AO}$ & 55: 5 & 60: 0 & 0.022 \\
\hline 5 View name & 55: 5 & 60: 0 & 0.022 \\
\hline \multicolumn{4}{|l|}{ ME 2C } \\
\hline $6 \mathrm{LA}$ & 53: 7 & 60: 0 & 0.006 \\
\hline $7 \mathrm{MV}$ & 53: 7 & 60: 0 & 0.006 \\
\hline $8 \mathrm{LV}$ & 51: 9 & 60: 0 & 0.002 \\
\hline 9 View name & $27: 33$ & 42: 28 & 0.006 \\
\hline \multicolumn{4}{|l|}{ ME RVOT } \\
\hline $10 \mathrm{LA}$ & 29: 31 & 43: 17 & 0.009 \\
\hline $11 \mathrm{RA}$ & 30: 30 & 43: 17 & 0.015 \\
\hline $12 \mathrm{TV}$ & 29: 31 & 42: 18 & 0.016 \\
\hline $13 \mathrm{RV}$ & $36: 24$ & 42: 18 & 0.251 \\
\hline $14 \mathrm{PV}$ & 28: 32 & 39: 21 & 0.043 \\
\hline $15 \mathrm{PA}$ & 28: 32 & 39: 21 & 0.043 \\
\hline $16 \mathrm{AV}$ & 45: 15 & 52: 8 & 0.104 \\
\hline 17 View name & 48: 12 & 52: 8 & 0.327 \\
\hline \multicolumn{4}{|l|}{ TG Basal SAX } \\
\hline $18 \mathrm{MV}$ & 51: 9 & 57: 3 & 0.068 \\
\hline
\end{tabular}

Data presented as the number for the sum of trainees who responded with correct or wrong interpretation of each anatomic structure, mean \pm standard deviation for the mean overall accuracy.

Abbreviations: ME Asc Aortic SAX (Middle esophagus ascend aortic short axis); ME Desc Aortic SAX (Middle esophagus descend aortic short axis); ME 2C (Middle esophagus two chamber); ME RVOT (Middle esophagus right ventricle inflow-outflow); TG Basal SAX (Transgastric basal short axis). 


\begin{tabular}{|llll|}
\hline Answers(correct:wrong) & Group V(n=60) & Group S( $\mathbf{n = 6 0 )}$ & P value \\
\hline 19View name & $51: 9$ & $57: 3$ & 0.068 \\
\hline Mean overall accuracy(\%) & $65.2 \pm 13.6 \%$ & $83.9 \pm 16.5$ & $<0.0001$ \\
\hline $\begin{array}{l}\text { Data presented as the number for the sum of trainees who responded with correct or wrong } \\
\text { interpretation of each anatomic structure, mean } \pm \text { standard deviation for the mean overall accuracy. }\end{array}$ \\
\hline $\begin{array}{l}\text { Abbreviations: ME Asc Aortic SAX (Middle esophagus ascend aortic short axis); ME Desc Aortic SAX } \\
\text { (Middle esophagus descend aortic short axis); ME 2C (Middle esophagus two chamber); ME RVOT } \\
\text { (Middle esophagus right ventricle inflow-outflow); TG Basal SAX (Transgastric basal short axis). }\end{array}$ \\
\hline
\end{tabular}


Table 5

Performance of Group V and Group S in the retention test 2.

\begin{tabular}{|c|c|c|c|}
\hline Answers(correct:wrong) & Group V(n=60) & Group $S(n=60)$ & $P$ value \\
\hline \multicolumn{4}{|l|}{ ME Desc Aortic SAX } \\
\hline $1 \mathrm{AO}$ & $60: 0$ & $60: 0$ & / \\
\hline 2 View name & $60: 0$ & $60: 0$ & / \\
\hline \multicolumn{4}{|l|}{ ME Desc Aortic LAX } \\
\hline $3 \mathrm{AO}$ & $60: 0$ & $60: 0$ & / \\
\hline 4 View name & $60: 0$ & $60: 0$ & / \\
\hline \multicolumn{4}{|l|}{ ME RVOT } \\
\hline $5 \mathrm{LA}$ & $26: 34$ & $33: 27$ & 0.201 \\
\hline $6 \mathrm{RA}$ & $31: 29$ & $38: 22$ & 0.196 \\
\hline $7 \mathrm{TV}$ & $30: 30$ & $37: 23$ & 0.198 \\
\hline $8 \mathrm{RV}$ & $36: 24$ & $37: 23$ & 0.852 \\
\hline $9 \mathrm{PV}$ & $27: 33$ & $30: 30$ & 0.583 \\
\hline $10 \mathrm{PA}$ & $27: 33$ & $32: 28$ & 0.361 \\
\hline $11 \mathrm{AV}$ & $51: 9$ & $55: 5$ & 0.255 \\
\hline 12 View name & $52: 8$ & $53: 7$ & 0.783 \\
\hline \multicolumn{4}{|l|}{ ME AV LAX } \\
\hline 13 LA & $43: 17$ & $58: 2$ & $<0.0001$ \\
\hline $14 \mathrm{MV}$ & $45: 15$ & $57: 3$ & 0.002 \\
\hline $15 \mathrm{LV}$ & $45: 15$ & $60: 0$ & $<0.0001$ \\
\hline $16 \mathrm{AV}$ & $59: 1$ & $60: 0$ & 0.315 \\
\hline $17 \mathrm{AO}$ & $59: 1$ & $60: 0$ & 0.315 \\
\hline $18 \mathrm{RV}$ & $28: 32$ & $36: 24$ & 0.143 \\
\hline 19 View name & $8: 52$ & $34: 26$ & $<0.0001$ \\
\hline
\end{tabular}

Data presented as the number for the sum of trainees who responded with correct or wrong interpretation of each anatomic structure, mean \pm standard deviation for the mean overall accuracy.

Abbreviations: ME Desc Aortic SAX (Middle esophagus descend aortic short axis); ME Desc Aortic LAX (Middle esophagus descend aortic long axis); ME RVOT (Middle esophagus right ventricle inflowoutflow); ME AV LAX (Middle esophagus aortic valve long axis); TG LAX (Transgastric long axis). 


\begin{tabular}{|llll|}
\hline Answers(correct:wrong) & Group V( $n=60)$ & Group $S(n=60)$ & P value \\
\hline TG LAX & & & \\
\hline 20 LA & $7: 53$ & $14: 46$ & 0.093 \\
\hline 21 MV & $5: 55$ & $11: 49$ & 0.107 \\
\hline 22 LV & $27: 33$ & $52: 8$ & $<0.0001$ \\
\hline 23 AV & $5: 55$ & $12: 48$ & 0.067 \\
\hline 24 AO & $5: 55$ & $11: 49$ & 0.107 \\
\hline 25 View name & $22: 38$ & $53: 7$ & $<0.0001$ \\
\hline Mean overall accuracy(\%) & $58.5 \pm 13.0$ & $71.5 \pm 10.9$ & $<0.0001$ \\
\hline $\begin{array}{l}\text { Data presented as the number for the sum of trainees who responded with correct or wrong } \\
\text { interpretation of each anatomic structure, mean } \pm \text { standard deviation for the mean overall accuracy. }\end{array}$ \\
\hline $\begin{array}{l}\text { Abbreviations: ME Desc Aortic SAX (Middle esophagus descend aortic short axis); ME Desc Aortic } \\
\text { LAX (Middle esophagus descend aortic long axis); ME RVOT (Middle esophagus right ventricle inflow- } \\
\text { outflow); ME AV LAX (Middle esophagus aortic valve long axis); TG LAX (Transgastric long axis). }\end{array}$ \\
\hline
\end{tabular}

\section{Discussion}

The results of the present study indicated that the students of Group S performed better in retention-test 1 and retention-test 2 for the interpretation of 20 cross-sectional views compared to the students of Group V.

The close contact of the transducer of TEE with the heart produced high-quality images in different views, allowing an easier and precise understanding of the three-dimensional heart. TEE is not only a diagnostic tool, rather a monitoring device during cardiac as well as noncardiac surgery[13].TEE can assist the anesthesiologists in evaluating the hemodynamic status of the patients, providing sufficient information to undertake decisions related to perioperative management, and improving patient outcomes[14, 15$]$. It is, therefore, essential and meaningful to train the anesthesiologists in the skills and knowledge regarding TEE[16]. Recent surveys indicated that only $46 \%$ of the critical care doctors received some kind of ultrasound training, and the percentage was even lower (27.5\%) among the anesthesiology residents[17, 18]. Moreover, the literature strongly recommends the introduction of ultrasound technology into medical education due to the ultrasound widespread and accessible nature of this technique and the benefit it provides to the patients, particularly its introduction in echocardiography training $[19,20]$.

The interpretation of 20 cross-sectional views requires the trainees to learn the normal cardiac anatomy, understand the orientation of the TEE probe, and clearly identify each anatomic structure in the echocardiographic image. First, using the simulator training system in the present study, the tutor simultaneously demonstrated the probe spatial orientation, the corresponding three-dimensional anatomic structures, and the two-dimensional echocardiographic images. The tutor manipulated the 
probe on the right screen while explaining how each corresponding echocardiographic view formed vividly on the left screen. The performances in the retention-tests 1 and 2 in Group S were higher than those in Group V, probably due to the hand-eye-head model used in the TEE simulator training system, which enabled the trainees to establish a correlation between the three-dimensional anatomic structures and the two-dimensional echocardiographic views in a short time, thereby assisting the participants to have deeper comprehensions rather than memorization only[11]. Second, when the participants began to manipulate the probe in the simulator, the knowledge from the lecture was stimulated and organized again in the participants' minds. The 30 min of practice on the dummy in Group S assisted the participants in putting the lecture theory and the tutor's demonstration into practice. Finally, the tutor supervised the participants' handling of the probe and assisted them in correcting the probe orientation and understanding the view interpretation whenever required. This correction and feedback on time assisted the participants in establishing a relationship between theory and practice. This approach of practice followed by a lecture is consistent with the constructivist theory, which emphasizes learning through a cycle of doing and reflecting[21]. While theory helped the participants practice better, the practice, in turn, enhanced the understanding of the theory, resulting in a "learning cycle"[22]. This theorypractice-theory cycle could have led to better learning and retention of TEE recognition.

Video-based training has been proven to serve as an adjunct for improving the interpretation of transthoracic echocardiographic (TTE) images among novice TTE trainers[23]. Ray et al. demonstrated that, despite a lack of live skills training, the knowledge of image interpretation was improved after watching a 30-min video comprising the three components of didactics (transthoracic echocardiographic views, including subcostal 4-chamber view, subcostal inferior vena cava view, parasternal long-axis view, para-sternal short-axis view, apical view, and pulmonary view), virtual practice, and clinical correlation[8]. In the present study also, the performance in the retention-test 1 was significantly higher than the performance in the post-test in Group V, which indicated that video-based training followed a lecture improved the knowledge of interpretation of TEE images. Even though video-based learning is considered an effective teaching method with ease of access, its acceptance is limited due to a lack of actual practice $[9,24]$. The video-based training conducted in our study, including image interpretation but no image acquisition practice, and it, therefore, lacked the hand-eye-head interaction and theory-practicetheory cycle, which might be the reason why the students did not have better retention of the images. Nonetheless, due to the advantages of efficiency, low cost, and ease of access, video-based training is suggested as a considerable choice when lacking a simulator, expert faculty, or sufficient practice time[7].

The performance in the retention-test 2 conducted one month later was significantly lower in both the groups compared to the performance in the retention-test 1 , which was conducted one week after the training, probably because the review of the obtained knowledge was not allowed in the present trial, indicating that regular review may help retain the complicated knowledge of TEE. In addition, training on actual patients has the potential to cause severe complications to the patients, particularly those under general anesthesia who unable to speak or move during the process of inserting and manipulating the probe, such as the patients with soft-tissue infection, postoperative dysphagia, digestive tract hemorrhage, etc. $[25,26]$. Using simulation at an early stage of learning for the novice trainees when the 
incidence of complications is the highest may effectively protect the patients from such risks[10]. However, even though the TEE simulator is considered an effective teaching tool for trainees, it has certain disadvantages. First, the TEE simulator is expensive and can be used only in a medical training center[27]. Second, the simulator may not satisfy the higher-level trainees as they are already proficient in the basic knowledge regarding TEE. For instance, when the trainees acquire the acceptable images, they may not know how to utilize the advanced features, such as spectral or color Doppler, using the simulator[28]. Third, a simulator cannot replace the clinical TEE practice at the bedside of actual patients as clinical TEE enables real-time monitoring and discovers any abnormal changes in the patient, which is not possible with the simulator.

\section{Limitations}

As with all research, the present study also had certain limitations. First, our study focused more on image interpretation rather than image acquisition. More advanced features, such as spectral or color Doppler were not focused on in our study. Second, the long-term retention test for the interpretation of 20 cross-sectional views was not conducted.

\section{Conclusions}

Following a 90-minute theoretical lecture, simulation-based training enhanced the performance of medical students in the interpretation and short-term retention of 20 cross-sectional views compared to video-based training.

\section{Abbreviations}

TEE: transesophageal echocardiographic;

TTE: transthoracic echocardiographic;

ME Asc Aortic SAX, Middle esophagus ascend aortic short axis;

ME Asc Aortic LAX, Middle esophagus ascend aortic long axis;

ME Desc Aortic SAX, Middle esophagus descend aortic short axis; ME Desc Aortic LAX, Middle esophagus descend aortic long axis;

UE Aortic Arch LAX, Upper esophagus aortic arch long axis;

UE Aortic Arch SAX, Upper esophagus aortic arch short axis;

ME 4C, Middle esophagus four chamber;

ME MC, Middle esophagus mitral commissural; 
ME 2C, Middle esophagus two chamber;

ME LAX, Middle esophagus long axis;

ME AV SAX, Middle esophagus aortic valve short axis;

ME RVOT, Middle esophagus right ventricle inflow-outflow;

ME Bicaval, Middle esophagus Bicaval;

ME AV LAX, Middle esophagus aortic valve long axis;

TG Basal SAX, Transgastric basal short axis;

TG Mid Papillary SAX, Transgastric middle papillary short axis view; TG 2C , Transgastric two-chamber;

TG LAX, Transgastric long axis;

TG RV inflow, Transgastric right ventricle inflow;

Deep TG LAX , Deep transgastric long axis $\rrbracket$

LA: left atrium;

LV: left ventricle;

MV: mitral valve;

AV: aortic valve;

RA: right atrium;

$\mathrm{RV}$ : right ventricle;

TV: tricuspid valve;

PV: pulmonary valve;

PA: pulmonary artery;

Group S: simulation-based group;

Group V: video-based group

\section{Declarations}

Ethics approval and consent to participate 
This was a single-center, randomized, double-blinded, prospective clinical trial study, which was registered in the Chinese registry of clinical trials at http://www.chictr.org.cn (ChiCTR2000033519, 3/June/2020).

The Research Ethics Committee of the Affiliated Hospital of North Sichuan Medical College approved the study (Approved No. 2020/111-1). This study adhered to the applicable CONSORT guidelines.

\section{Consent for publication}

Not applicable to our manuscript.

\section{Availability of data and materials}

We declared that materials described in the manuscript, including all relevant raw data, will be freely available to any scientist wishing to use them for non-commercial purposes, without breaching participant confidentiality. Y.Z.(594624370@qq.com) would be contacted if someone wants to request the data.

\section{Competing interests}

The authors declare no competing interests.

\section{Funding}

This research was funded by the project of Affiliated hospital North Sichuan Medical College (grant no. 2016-163) which belongs to Y.Z. The funding supports the publish payment of the study. Disclosure: None.

\section{Authors' contributions:}

T.-Z. and Y.Z are responsible for the idea and design of the study.

J.-Y.L did the randomization and quality control of this study.

Y.Z. and D.Q taught the lecture and TEE training.

H.-Y.Z. and Z.-Y.Y. did the tests for the whole period.

X-Y. and H-B.S did the statistical analysis.

Y.Z and Z.-Y.Y. drafted and T.-Z. revised the article.

All authors read and approved the final manuscript.

\section{Authors' information}

Tao Zhu: professor, Ph.D., doctoral supervisor, vice chief of the department of anesthesiology, West China Hospital of Sichuan University, Chengdu, 610041, Sichuan, P.R. China. Major in anesthesiology, 
perioperative organ protection, mechanism of anesthetic drugs, and perioperative information management. He has published over $30 \mathrm{SCl}$ papers as first author or corresponding author.

\section{Acknowledgments}

The authors gratefully acknowledge the Department of Anesthesiology at the West China Hospital of Sichuan University and Chengdu Branch of the Chinese Academy of Sciences provide the VirSim TEE training system, the third-generation TEE simulator for our study.

\section{References}

1. American Society of A, Society of Cardiovascular Anesthesiologists Task Force on Transesophageal E: Practice guidelines for perioperative transesophageal echocardiography. An updated report by the American Society of Anesthesiologists and the Society of Cardiovascular Anesthesiologists Task Force on Transesophageal Echocardiography. Anesthesiology 2010, 112(5):1084-1096.

2. Barber RL, Fletcher SN: A review of echocardiography in anaesthetic and peri-operative practice. Part 1: impact and utility. Anaesthesia 2014, 69(7):764-776.

3. Skinner $\mathrm{H}$, Morgan-Hughes $\mathrm{N}$, Swanevelder J, More R: Accreditation in transoesophageal echocardiography in the UK: the initial experience. Br J Anaesth 2012, 109(4):487-490.

4. Shanewise JS, Cheung AT, Aronson S, Stewart WJ, Weiss RL, Mark JB, Savage RM, Sears-Rogan P, Mathew JP, Quinones MA et al: ASE/SCA guidelines for performing a comprehensive intraoperative multiplane transesophageal echocardiography examination: recommendations of the American Society of Echocardiography Council for Intraoperative Echocardiography and the Society of Cardiovascular Anesthesiologists Task Force for Certification in Perioperative Transesophageal Echocardiography. Anesth Analg 1999, 89(4):870-884.

5. Antiel RM, Van Arendonk KJ, Reed DA, Terhune KP, Tarpley JL, Porterfield JR, Hall DE, Joyce DL, Wightman SC, Horvath KD et al: Surgical training, duty-hour restrictions, and implications for meeting the Accreditation Council for Graduate Medical Education core competencies: views of surgical interns compared with program directors. Arch Surg 2012, 147(6):536-541.

6. Mitchell JD, Mahmood F, Bose R, Hess PE, Wong V, Matyal R: Novel, multimodal approach for basic transesophageal echocardiographic teaching. J Cardiothorac Vasc Anesth 2014, 28(3):800-809.

7. Edrich T, Stopfkuchen-Evans M, Scheiermann P, Heim M, Chan W, Stone MB, DankI D, Aichner J, Hinzmann D, Song P et al: A Comparison of Web-Based with Traditional Classroom-Based Training of Lung Ultrasound for the Exclusion of Pneumothorax. Anesth Analg 2016, 123(1):123-128.

8. Ray JJ, Meizoso JP, Hart V, Horkan D, Behrens V, Rao KA, Karcutskie CA, Lenchus J, Schulman Cl, Dudaryk R: Effectiveness of a Perioperative Transthoracic Ultrasound Training Program for Students and Residents. J Surg Educ 2017, 74(5):805-810.

9. Gradl-Dietsch G, Menon AK, Gursel A, Gotzenich A, Hatam N, Aljalloud A, Schrading S, Holzl F, Knobe $\mathrm{M}$ : Basic echocardiography for undergraduate students: a comparison of different peer-teaching 
approaches. Eur J Trauma Emerg Surg 2018, 44(1):143-152.

10. Harrison CM, Gosai JN: Simulation-based training for cardiology procedures: Are we any further forward in evidencing real-world benefits? Trends Cardiovasc Med 2017, 27(3):163-170.

11. Song H, Peng YG, Liu J: Innovative transesophageal echocardiography training and competency assessment for Chinese anesthesiologists: role of transesophageal echocardiography simulation training. Curr Opin Anaesthesio/ 2012, 25(6):686-691.

12. Thampi S, Lee CCM, Agrawal RV, Ashokka B, Ti LK, Paranjothy S, Ponnamperuma GG: Ideal Sequence of Didactic Lectures and Simulation in Teaching Transesophageal Echocardiography Among Anesthesiologists. J Cardiothorac Vasc Anesth 2020, 34(5):1244-1249.

13. Mahmood F, Christie A, Matyal R: Transesophageal echocardiography and noncardiac surgery. Semin Cardiothorac Vasc Anesth 2008, 12(4):265-289.

14. Schulmeyer MC, Santelices E, Vega R, Schmied S: Impact of intraoperative transesophageal echocardiography during noncardiac surgery. J Cardiothorac Vasc Anesth 2006, 20(6):768-771.

15. Shillcutt SK, Markin NW, Montzingo CR, Brakke TR: Use of rapid "rescue" perioperative echocardiography to improve outcomes after hemodynamic instability in noncardiac surgical patients. J Cardiothorac Vasc Anesth 2012, 26(3):362-370.

16. Goldstein S: Pro: the general anesthesiologist should be trained and certified in transesophageal echocardiography. J Cardiothorac Vasc Anesth 2010, 24(1):183-188.

17. Mosier JM, Malo J, Stolz LA, Bloom JW, Reyes NA, Snyder LS, Adhikari S: Critical care ultrasound training: a survey of US fellowship directors. J Crit Care 2014, 29(4):645-649.

18. Conlin F, Roy Connelly N, Raghunathan K, Friderici J, Schwabauer A: Focused Transthoracic Cardiac Ultrasound: A Survey of Training Practices. J Cardiothorac Vasc Anesth 2016, 30(1):102-106.

19. Bahner DP, Goldman E, Way D, Royall NA, Liu YT: The state of ultrasound education in U.S. medical schools: results of a national survey. Acad Med 2014, 89(12):1681-1686.

20. Soucy ZP, Mills LD: American Academy of Emergency Medicine Position Statement: Ultrasound Should Be Integrated into Undergraduate Medical Education Curriculum. J Emerg Med 2015, 49(1):89-90.

21. Wong A: Review article: teaching, learning, and the pursuit of excellence in anesthesia education. Can $J$ Anaesth 2012, 59(2):171-181.

22. Issenberg SB, McGaghie WC, Petrusa ER, Lee Gordon D, Scalese RJ: Features and uses of highfidelity medical simulations that lead to effective learning: a BEME systematic review. Med Teach 2005, 27(1):10-28.

23. Rebel A, Srour H, DiLorenzo A, Nguyen D, Ferrell S, Dwarakanatli S, Haas E, Schell RM: Ultrasound Skill and Application of Knowledge Assessment using an Innovative OSCE Competition-Based Simulation Approach. J Educ Perioper Med 2016, 18(1):E404.

24. Weber U, Constantinescu MA, Woermann U, Schmitz F, Schnabel K: Video-based instructions for surgical hand disinfection as a replacement for conventional tuition? A randomised, blind 
comparative study. GMS J Med Educ 2016, 33(4):Doc57.

25. Hahn RT, Abraham T, Adams MS, Bruce CJ, Glas KE, Lang RM, Reeves ST, Shanewise JS, Siu SC, Stewart W et al: Guidelines for performing a comprehensive transesophageal echocardiographic examination: recommendations from the American Society of Echocardiography and the Society of Cardiovascular Anesthesiologists. J Am Soc Echocardiogr 2013, 26(9):921-964.

26. Kim HY, Lee SC, Park SJ, Choi JO, Chang SA, Kim SM, Choe YH, Oh JK, Park SW: A Rare Case of latrogenic Deep Neck Infection Secondary to Hypopharyngeal Injury Caused by the Transesophageal Echocardiography. J Cardiovasc Ultrasound 2015, 23(3):181-185.

27. Bose R, Matyal R, Panzica P, Karthik S, Subramaniam B, Pawlowski J, Mitchell J, Mahmood F: Transesophageal echocardiography simulator: a new learning tool. $J$ Cardiothorac Vasc Anesth 2009, 23(4):544-548.

28. Smith WB, Robinson AR, 3rd, Janelle GM: Expanding role of perioperative transesophageal echocardiography in the general anesthesia practice and residency training in the USA. Curr Opin Anaesthesio/ 2015, 28(1):95-100.

\section{Figures}




Enrollment

Randomization

\section{Analysis}

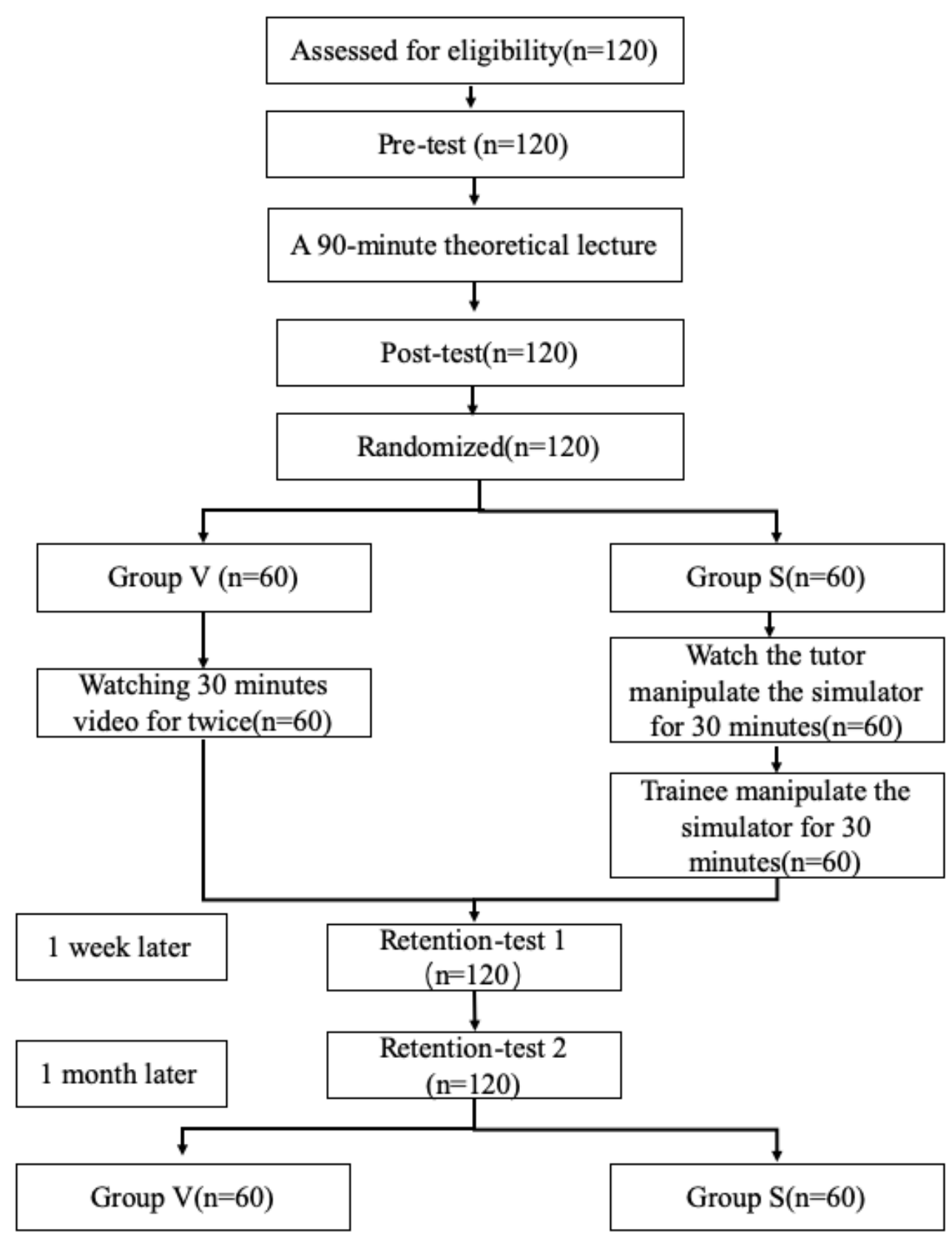

\section{Figure 1}

Flow chart 


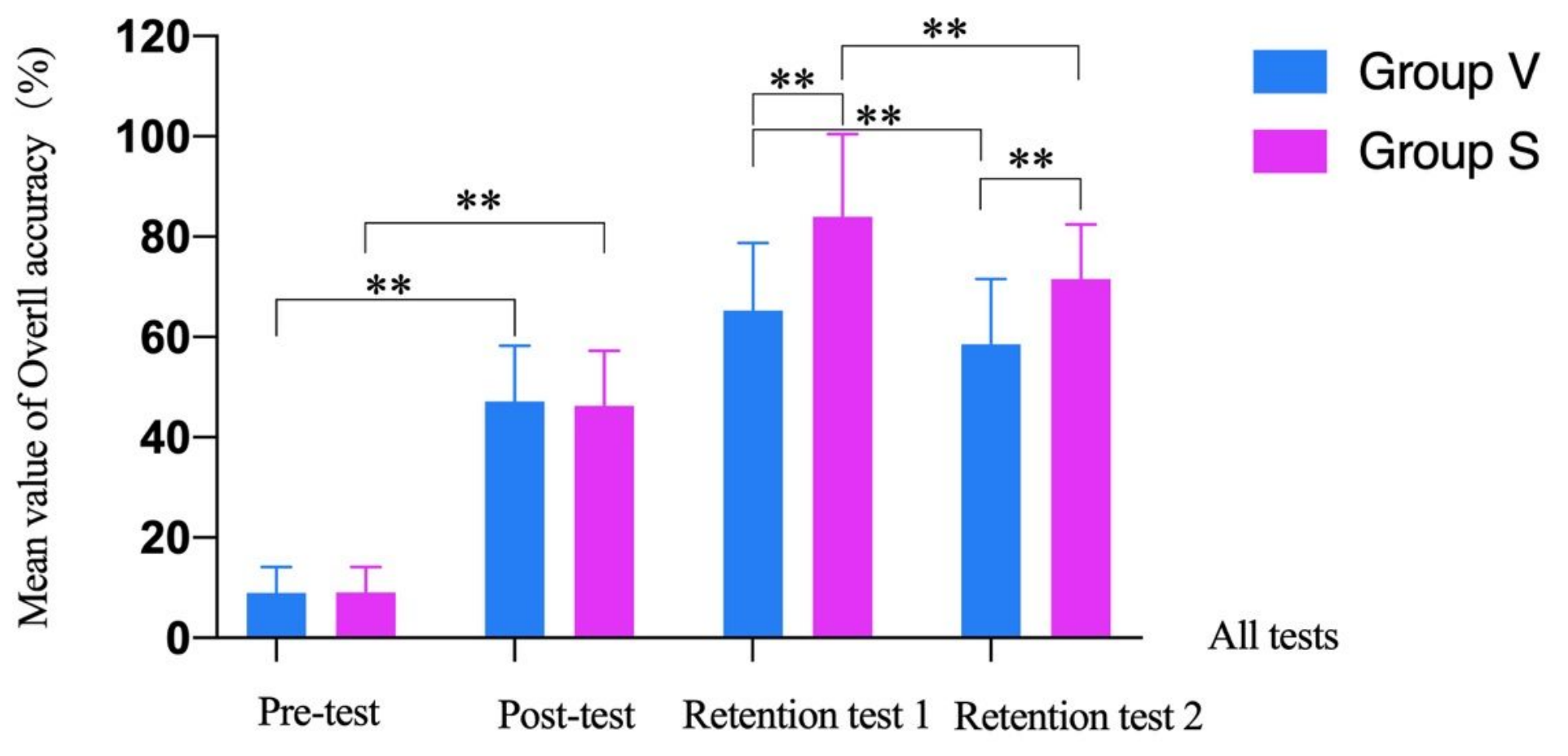

Figure 2

The comparison of the two groups in all the tests 